\title{
Role of Mitochondrial DNA in Cells Exposed to Irradiation: Generation of Reactive Oxygen Species (ROS) is Required for G2 Checkpoint upon Irradiation
}

\author{
Saori Kawamura, ${ }^{a}$ Daisaku Takai, ${ }^{a}$ Keiko Watanabe, ${ }^{a}$ Jun-ichi Hayashi, ${ }^{b}$ Kazushige Hayakawa, ${ }^{c}$ \\ and Makoto Akashi*,a \\ ${ }^{a}$ Department of Radiation Emergency Medicine, Research Center for Radiation Emergency Medicine, National Institute of Radiological \\ Sciences, 4-9-1 Anagawa, Inage-ku, Chiba 263-8555, Japan, ' Institute of Biological Sciences, University of Tsukuba, 1-1-1 Tennodai, \\ Tsukuba, Ibaraki 305-8572, Japan, and 'Department of Radiology, Kitasato University School of Medicine, 1-15-1 Kitasato, \\ Sagamihara, Kanagawa 228-8555, Japan
}

(Received February 4, 2005; Accepted February 7, 2005)

\begin{abstract}
Mitochondria have their own genome encoding subunits of the electron transport chain. Using cells lacking mitochondrial DNA (mtDNA, $\rho^{0}$ cells), we studied the role of mtDNA in irradiation. Loss of mtDNA inhibited cell growth and reduced the level of reactive oxygen species (ROS) as compared to $\rho^{+}$cells. $\rho^{+}$cells were more resistant to irradiation than $\rho^{0}$ cells. Upon irradiation, $\rho^{0}$ cells showed delayed G2 arrest and decreased ability of a cell to recover from the G2 checkpoint compared to $\rho^{+}$cells. Irradiation increased the generation of ROS even more in $\rho^{+}$cells. Irradiation markedly increased the levels of phosphorylated forms of extracellular-regulated kinases, p42 and p44 (ERK1/2) in $\rho^{+}$cells, whereas phosphorylated levels of the kinases were affected slightly in $\rho^{0}$ cells. Furthermore, inhibition of the ERK pathway led to a delayed G2 arrest and a delayed recovery from the arrest in irradiated $\rho^{+}$cells, and treatment with NAC also induced dysfunction of the $\mathrm{G} 2$ checkpoint in irradiated $\rho^{+}$cells. These results suggest that the accumulation of ROS potentiated ERK1/2 kinases after irradiation in $\rho^{+}$cells, leading to less sensitivity to irradiation. Thus, mtDNA is important for the generation of ROS that act as second messenger.
\end{abstract}

Key words - mitochondrial DNA, irradiation, reactive oxygen species, checkpoint

\section{INTRODUCTION}

Mitochondria have their own DNA (mtDNA) of

*To whom correspondence should be addressed: Department of Radiation Emergency Medicine, Research Center for Radiation Emergency Medicine, National Institute of Radiological Sciences, 4-9-1 Anagawa, Inage-ku, Chiba 263-8555 Japan. Tel.: +81-43-206-3122; Fax: +81-43-284-1736; E-mail: akashi@nirs.go.jp
16 kilo base pairs in human cells. mtDNA encodes subunits of the mitochondrial electron transport chain. These subunits are essential for normal oxidative phosporylation and also adenosine triphosphate (ATP) production in a cell. Oxidative phosphorylation in mitochondria produces the large part of the energy required within a cell. ${ }^{1)}$ Human mtDNA has a mutational rate at least 10 times higher than nuclear DNA, ${ }^{2)}$ and somatic mutations in mtDNA have also been observed in human neoplasms. ${ }^{3)} \mathrm{Re}-$ cently, there have been reports using cells lacking mtDNA that studied its roles in cell death or sensitivity to various external insults. Studies have shown that mtDNA-depleted cells are susceptible to cell death by serum-deprivation, tumor necrosis factor (TNF) and staurosporine. ${ }^{4-6}$ On the other hand, studies found that these cells were more resistant to insults such as tumor necrosis factor-related apoptosisinducing ligand (TRAIL), anti-cancer reagents, and reactive oxygen species (ROS) than their parental cells. ${ }^{7,8}$ Thus, the roles of mtDNA are still not clear, and the mechanisms for the sensitivity to external insults remain unresolved.

The respiratory chain in mitochondria is a source of ROS..$^{9-12)}$ On the other hand, studies have shown that ROS generated in mitochondria can modulate signaling cascades. ${ }^{13-15)}$ The pathway of the mitogen-activated protein kinase (MAPK) family is involved in growth factor-mediated regulation of diverse cellular events such as proliferation, senescence, differentiation and apoptosis. ${ }^{16)}$ Exposure of cells to oxidative stress such as irradiation induces activation of multiple MAPK pathways; these signals play critical roles in controlling cell survival and repopulation effects following irradiation. ${ }^{16)}$ The MAPK superfamily is composed of several signal- 
ing pathways: mitogen-activated extracellular-regulated kinases, p42 and p44 (ERK1/2), c-Jun N-terminal kinase (JNK), and p38 pathways. ERK1/2 are serine/threonine kinases that are regulated by mitogen-activated/extracellular-regulated kinase 1/2 (MEK1/2) through phosphorylation. The activation of ERK1/2 protects cells against noxious stimuli in several ways and inhibits apoptosis through the activation of caspase or the inhibition of cytochrome $\mathrm{c}$ release. ${ }^{17,18)}$ Furthermore, ERK1/2 activation has been reported to promote several transcriptional factors such as nuclear factor-kappa B (NF- $\kappa \mathrm{B})$ and cyclic adenosine monophosphate responsive element binding protein (CREB), which then stimulate the expression of anti-apoptotic genes. ${ }^{19)}$ The activation of ERK1/2 has been reported to abrogate the $\mathrm{G} 2 / \mathrm{M}$ phase arrest after irradiation in some cell types. $^{20,21)}$ In contrast, JNK and p38 MAPK have been reported to mediate caspase activation, resulting in apoptosis. ${ }^{16)}$ However, the roles of mtDNA and how ROS generated in mitochondria affect signaling pathways are not fully understood in cells exposed to irradiation.

The recent progress in studies of mitochondria has allowed us to use mtDNA-depleted cells ( $\rho^{0}$ cells) and their control cells $\left(\rho^{+}\right.$cells). Elimination of mtDNA from cells can be performed by long-term exposure to a low concentration of 3,8-diamino-5ethyl-6-phenylphenanthridinium bromide (ethidium bromide; EtBr). ${ }^{22)} \rho^{+}$cells are produced by the fusion of cytoplasts from the parent cells with $\rho^{0}$ cells, and these cells possess normal mtDNA. By comparing $\rho^{0}$ to $\rho^{+}$cells, the roles of mtDNA can be determined. Irradiation is well known to induce apoptosis and cause DNA damage. However, there are few studies on the roles of mtDNA in irradiated cells. In the present study, we investigated the roles of mtDNA in irradiated cells by comparing $\rho^{0}$ cells lacking mtDNA and their control $\rho^{+}$cells. We found that $\rho^{0}$ cells were more radiosensitive than $\rho^{+}$cells, and demonstrated that homeostatic mitochondrial ROS production may have a protective effect on cells exposed to irradiation through MEK activation and cell cycle control.

\section{MATERIAL AND METHODS}

Cells and Cell Culture —Cells used in this study originated from HeLa cells: $\rho^{0}$ cells (EB8-C) lacking mtDNA, and $\rho^{+}$cells (HeEB5) cybrid clones produced by fusion of EB8-C cells and cytoplasts of
HeLa cells. ${ }^{22,23)}$ For cell culture, the medium consisted of RPMI 1640 (Gibco, Invitrogen Corp., CA, U.S.A.) supplemented with $10 \%(\mathrm{v} / \mathrm{v})$ fetal bovine serum (INTERGEN, Purchase, NY, U.S.A.), $50 \mu \mathrm{g} /$ $\mathrm{ml}$ of uridine (Sigma, St. Louis, MO, U.S.A.) and $1 \mathrm{mM}$ of sodium pyruvate (Gibco).

Polymerase Chain Reaction (PCR) — Total DNA was extracted by the phenol/chloroform method. Primers were specific for the segment of mitochondrial DNA: forward, $5^{\prime}$-atg ccc caa cta aat act acc g- $3^{\prime}$ and reverse, $5^{\prime}$-gtg gtg att agt cgg ttg ttg a-3'. After electrophoresis on 2\% agarose, the 298-bp fragment was analyzed with ethidium bromide (Sigma) under UV light.

Clonogenic Assays — For colony formation assay, $10^{2}$ to $5 \times 10^{3}$ cells were seeded in $60-\mathrm{mm}$ culture plates $18 \mathrm{hr}$ before irradiation at various doses. Colonies were stained and counted after 14 days; groups of cells containing at least 50 cells were defined as a colony. Colonies with diameter greater than $1.2 \mathrm{~mm}$ were defined as "larger colonies" using Intelligent Quantifier ${ }^{\mathrm{TM}}$ (Bio Image Systems, Inc., Jackson, MI, U.S.A.). Survival curves are representative data from three independent experiments. Radiation Setting $-{ }^{137} \mathrm{Cs}$ source emitting at a fixed dose rate of $10 \mathrm{~Gy} / \mathrm{min}$ was used for $\gamma$-ray irradiation.

Cell Cycle Analysis — After irradiation, cells were fixed with $70 \%$ ethanol at the times indicated. Then the cells were incubated for $30 \mathrm{~min}$ at $37^{\circ} \mathrm{C}$ with $1 \mu \mathrm{g} / \mathrm{ml} \mathrm{RNase} \mathrm{A,} \mathrm{stained} \mathrm{with} 50 \mu \mathrm{g} / \mathrm{ml}$ propidium iodide and filtered through nylon mesh with a pore size of 50-70 $\mu \mathrm{m}$. Cell cycle profiles were evaluated by FACScaliber flow cytometry (Becton Dickinson, Bedford, MA, U.S.A.).

\section{Analysis of Intracellular ROS Production}

$2^{\prime}, 7^{\prime}$-dichlorofluorescein (DCF) is one of the most prevalent oxidant-sensitive fluorescent dye. Acetyl ester derivative of dihydro-DCF diacetate (CM$\mathrm{H}_{2}$ DCFDA, Molecular Probes, Eugene, OR, U.S.A.), a derivative of DCF, was used in this study. Cells were prepared $18 \mathrm{hr}$ before the treatment to a cell count of $6 \times 10^{5}$ for each 60 -mm diameter dish. After irradiation, cells were stained with $10 \mu \mathrm{M} \mathrm{CM}$ $\mathrm{H}_{2}$ DCFDA at $37^{\circ} \mathrm{C}$ for $5 \mathrm{~min}$. Fluorescence intensity was measured by flow cytometry with excitation at $488 \mathrm{~nm}$ and emission at $530 \mathrm{~nm}$.

Western-Blot Analysis — The cells were lysed in buffer containing $50 \mathrm{mM}$ Tris- $\mathrm{HCl}(\mathrm{pH} \mathrm{8.0)}$, $150 \mathrm{mM} \mathrm{NaCl}, 3 \mathrm{mM} \mathrm{NaN}, 0.1 \%$ (w/v) sodium dodecyl sulfate (SDS), $1 \%$ (v/v) Nonidet P-40 and $0.5 \%$ sodium deoxycholate. The protein concentra- 
tion of each sample was measured by the method of Bradford (Bio-Rad, Hercules, CA, U.S.A.). ${ }^{24)}$ Electrophoresis was performed with samples containing $50 \mu \mathrm{g}$ of cell lysates in SDS-polyacrylamide gel electrophoresis (PAGE) loading buffer using 10\% polyacrilamide gel, followed by transfer of the proteins onto pure nitrocellulose membranes (TransBlot Transfer Membrane; Bio-Rad). Immunoreactivity was detected by enhanced chemiluminescence (Amersham Biosciences). The relative density of bands was determined by Intelligent Quantifier program (Bio Image, Ann Arbor, MI, U.S.A.) and normalized to the loading control.

\section{RESULTS}

\section{$\rho^{0}$ Cells Lacking mtDNA}

$\rho^{0}$ cells lacking mtDNA (named HeLa EB8-C) were made by long-term EtBr treatment. ${ }^{23)} \rho^{+}$cells (HeEB5) were cybrids of EB8-C cells with intact mtDNA. To confirm the absence of mtDNA, the 298-bp segment of mtDNA was amplified by polymerase chain reaction (PCR) analysis (Fig. 1). No detectable band corresponding to mtDNA was noted in EB8-C cells, whereas clear bands were amplified in HeEB5 cells. We also confirmed the absence of mtDNA by the failure to grow in the absence of uridine in the medium, since $\rho^{0}$ cells are dependent on uridine and pyruvate for growth because of the absence of a functional respiratory chain ${ }^{22}$ ) (data not shown). Thus, EB8-C cells were proven to be defective in oxidative phosphorylation and were used in the present study.

\section{Lower Production of ROS in EB8-C Cells}

CM- $\mathrm{H}_{2}$ DCFDA was used for experiments because of its better retention within cells than DCF. We compared ROS generation in HeEB5 and EB8$\mathrm{C}$ cells by flow cytometry. The mean fluorescence intensities of untreated HeEB5 and EB8-C cells were $51 \pm 4$ and $26 \pm 2$, respectively, indicating that HeEB 5 cells produced ROS constitutively at a higher level than EB8-C cells $(p<0.005)$.

The ROS production is known to increase after irradiation in cells. ${ }^{12,25)}$ The generation of ROS was evaluated at $30 \mathrm{~min}$ after irradiation in the two cell lines. Exposure to irradiation with $20 \mathrm{~Gy}$ resulted in significantly increased fluorescence intensity in HeEB5 cells (136 $\pm 10,2.6$-fold, $p<0.01)$, but the fluorescent intensities were not increased in EB8-C cells $(23 \pm 1)$.

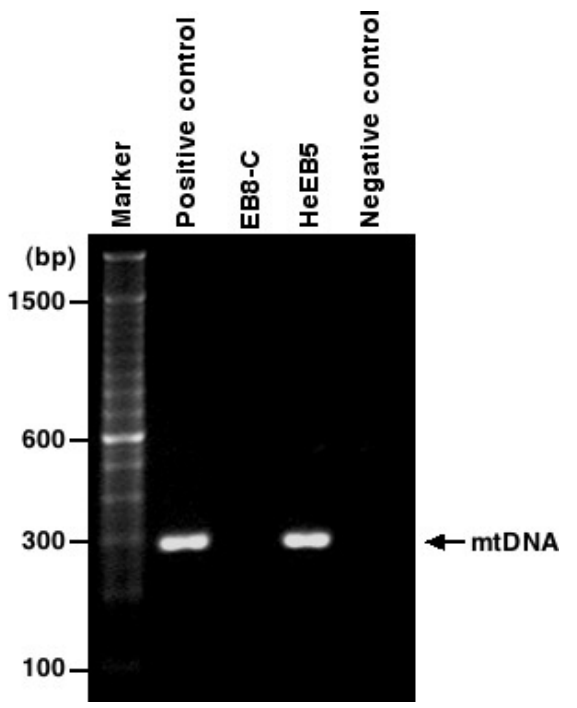

Fig. 1. Comparison between EB8-C and HeEB5 Cells mtDNA was amplified by PCR as described in MATERIALS AND METHODS. The arrow indicates 298-bp fragments of mtDNA. SK HEP-1 hepatocellular carcinoma cells were used as positive control. As negative control, purified water was used.

The specificity of ROS was also determined by irradiation in the presence of catalase from human erythrocytes (Calbiochem, La Jolla, CA, U.S.A.) in HeEB5 cells. The treatment with catalase abolished the irradiation-induced increase in the fluorescent intensity (data not shown). Furthermore, irradiated HeEB5 cells were stained with dihydroethidium (DHE, Molecular Probes) specific for superoxide radicals. However, an increased intensity of fluorescence was not detected in these cells (data not shown). These results suggest that irradiation increased the level of ROS, mainly hydrogen peroxide in HeEB5 cells.

\section{Increased Sensitivity of EB8-C Cells to Irradia- tion}

Next we sought to determine whether the observed difference in ROS production affects the survival and growth of cells after irradiation. Cellular radiosensitivity was analyzed by colony formation assay. The plating efficiencies were $87.7 \pm 2.5$ and $50.3 \pm 4.5 \%$ in HeEB5 cells and EB8-C cells, respectively, and the efficiency was significantly reduced in EB8-C cells $(p<0.001)$. Further study found a significantly reduced survival fraction in EB8-C irradiated with 2 Gy (Fig. 2). The surviving fractions were $0.33 \pm 0.01$ and $0.19 \pm 0.03$ in HeEB cells and EB8-C cells, respectively $(p<0.01)$. At 4 Gy of irradiation, the fractions were $0.21 \pm 0.02$ for HeEB5 cells and $0.06 \pm 0.00$ for EB8-C cells, 
showing a significantly decreased survival fraction in EB8-C cells compared to HeEB5 cells ( $p<0.005$ ). Upon irradiation with either 6 or $8 \mathrm{~Gy}$, significant differences in sensitivity to irradiation were observed between these cell lines.

To further evaluate the cell growth after irradiation, the diameter of the colonies was quantitatively evaluated (Table 1). HeEB5 cells had a significantly higher capacity for forming colonies larger than $1.2 \mathrm{~mm}$ in diameter than EB8-C cells. The capacity for forming larger colonies was $15.1 \pm 1.7 \%$ in the control. On the other hand, $5.5 \pm 0.2 \%$ of untreated EB8-C cells formed larger colonies. HeEB5 cells also formed larger colonies than EB8-C cells at each dose of irradiation. At $1 \mathrm{~Gy}, \mathrm{HeEB} 5$ cells had $14.7 \pm$ $3.1 \%$ of the capacity, whereas that of EB8-C cells was $1.8 \pm 0.4 \%$. Thus, depleting mtDNA affected cell growth and resulted in an increased sensitivity to irradiation.

\section{Dysfunction of the G2 Checkpoint in Irradiated EB8-C Cells}

Cells have mechanisms to delay or halt cell cycle progression in response to genotoxic insult to maintain genomic integrity. ${ }^{26)} \mathrm{HeLa}$ cells are known to be infected with human papilloma virus, of which the E6 protein inactivates p53; the checkpoint may

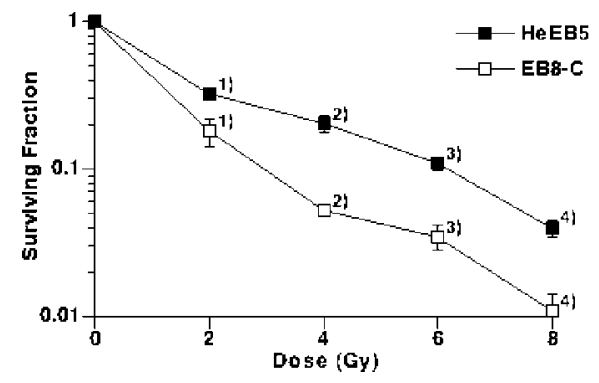

Fig. 2. Radiosensitivity of Cells Lacking mtDNA

HeEB5 and EB8-C cells were exposed to ionizing radiation at indicated doses. After two weeks, colonies were stained with hematoxilineosin and counted. All experiments were triplicated. The results were the mean of three independent experiments. 1) $p<0.01,2-4) p<0.005$. not function in cells exposed to ionizing radiation. ${ }^{27)}$ To determine whether such checkpoint mechanisms are associated with the observed sensitivity to irradiation in EB8-C cells, cell cycle progression after irradiation was studied (Table 2). Without irradiation, the cell cycle profiles of both cell lines were similar. After irradiation with $8 \mathrm{~Gy}, 60 \%$ of HeEB5 cells had entered the G2 phase by $12 \mathrm{hr}$. Thereafter, the cells gradually re-entered G1 and then S phase by $36 \mathrm{hr}$. On the other hand, the cell cycle progression was delayed after irradiation in EB8-C cells when compared to HeEB5 cells. Cells gradually entered the $\mathrm{G} 2$ phase by $24 \mathrm{hr}$, and $60 \%$ of cells remained at $\mathrm{G} 2$ phase $36 \mathrm{hr}$ after irradiation. Thus, EB8-C cells showed delayed induction of G2 arrest and decreased ability to recover from $\mathrm{G} 2$ arrest.

\section{Activation of ERK Pathway in Irradiated HeEB5 Cells}

Previously, we have shown that MAPK is involved in the sensitivity to irradiation. ${ }^{28)}$ To study the mechanism causing the different sensitivity to irradiation in the two cell lines, the MAPK pathway was determined. Cells were irradiated at a dose of 8 Gy and cultured for $1 \mathrm{hr}$. Western blot analysis using antibody recognizing the phosphorylated form of ERK1/2 showed that it was more abundant in untreated HeEB5 cells than in untreated EB8-C cells; EB8-C cells had a faint band of phosphorylated form of ERK1/2 (Fig. 3). HeEB5 cells had almost 4-fold higher level of phosphorylated form of ERK1/2 as compared to that of EB8-C cells. Irradiation activated ERK1/2 by 2-fold in HeEB5 cells. However, irradiation failed to increase the level of phosphorylated form of ERK1/2 in EB8-C cells. Thus, the activation of the ERK pathway was much more prominent in HeEB5 cells than in EB8-C cells. We also studied the effect of irradiation on the phosphorylation of p38MAPK in these cells. However, phosphorylation of p38MAPK was not induced in both cell lines (data not shown).

Table 1. Numbers of Colonies Larger than $1.2 \mathrm{~mm}$ after Irradiation in HeEB5 and EB8-C Cells

\begin{tabular}{lrrrrrr}
\hline \hline & \multicolumn{6}{c}{ Percentage of larger colonies } \\
\cline { 2 - 6 } & \multicolumn{1}{c}{0} & \multicolumn{6}{c}{ 1 } & 2 & 4 & 6 & $8(\mathrm{~Gy})$ \\
\hline HeEB5 & $15.1 \pm 1.7^{a)}$ & $14.7 \pm 3.1^{b)}$ & $11.9 \pm 1.1^{c)}$ & $8.2 \pm 1.2^{d)}$ & $6.4 \pm 0.5^{e)}$ & $5.8 \pm 0.9^{f)}$ \\
EB8-C & $5.5 \pm 0.2^{a)}$ & $1.8 \pm 0.4^{b)}$ & $1.4 \pm 0.4^{c)}$ & $1.1 \pm 0.0^{d)}$ & $0.2 \pm 0.1^{e)}$ & $2.0 \pm 0.2^{f)}$ \\
\hline
\end{tabular}

Cells were irradiated at indicated doses and the numbers of colonies $(>1.2 \mathrm{~mm}$ in diameter) were calculated as described in "MATERIALS AND METHODS." All experiments were triplicated and representative results of three independent experiments are shown as mean \pm S.D. a) $p<0.01, b, d, f) p<0.05, c, e) p<0.005$. 
Table 2. Cell Cycle Profile after Irradiation in HeEB5 and EB8-C Cells

\begin{tabular}{lllllll}
\hline \hline & \multicolumn{5}{c}{ Distribution of Cells in Each Stage (\%) } \\
\cline { 2 - 6 } & Stage & $0 \mathrm{hr}$ & $8 \mathrm{hr}$ & $12 \mathrm{hr}$ & $24 \mathrm{hr}$ & $36 \mathrm{hr}$ \\
\hline HeEB5 & & & & & & \\
Untreated & $\mathrm{G}_{1}$ & 46 & 51 & 52 & 54 & 54 \\
& $\mathrm{~S}$ & 35 & 30 & 32 & 30 & 31 \\
& $\mathrm{G}_{2} / \mathrm{M}$ & 19 & 19 & 16 & 16 & 15 \\
IR (8 Gy) & $\mathrm{G}_{1}$ & & 19 & 6 & 48 & 42 \\
& $\mathrm{~S}$ & & 46 & 34 & 9 & 36 \\
& $\mathrm{G}_{2} / \mathrm{M}$ & & 35 & 60 & 42 & 22 \\
EB8-C & & & & & & \\
Untreated & $\mathrm{G}_{1}$ & 55 & 57 & 55 & 55 & 59 \\
& $\mathrm{~S}$ & 31 & 38 & 30 & 31 & 30 \\
& $\mathrm{G}_{2} / \mathrm{M}$ & 14 & 15 & 15 & 14 & 11 \\
IR (8 Gy) & $\mathrm{G}_{1}$ & & 31 & 19 & 3 & 35 \\
& $\mathrm{~S}$ & & 50 & 49 & 9 & 5 \\
& $\mathrm{G}_{2} / \mathrm{M}$ & & 19 & 32 & 88 & 60 \\
\hline
\end{tabular}

HeEB5 and EB8-C cells were irradiated at a dose of $8 \mathrm{~Gy}$ and harvested at the times indicated. Cells were fixed with $70 \%$ ethanol and treated with RNase A. Then, cells were stained with propidium iodide (PI) and the DNA content was analyzed by flow cytometry using CellQuest and Modifit programs.

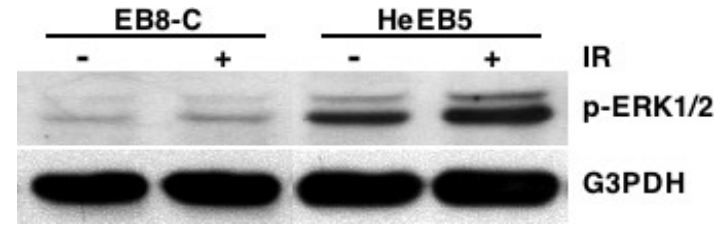

Fig. 3. Expression of ERK in Cells Exposed to Ionizing Radiation

Cells were incubated for an hour after irradiation with $8 \mathrm{~Gy}$. Whole cell lysates were used for immunoblotting with anti-phosphorylated ERK1/2 antibody (Thr202/Tyr204, Cell Signaling Technology, Inc. Beverly, MA, U.S.A.). As loading control, glyceroaldehyde-3-phosphate dehydrogenase (G3PDH) was used. The results are representative of three independent experiments.

\section{Inhibition of ERK Pathway Results in Dysfunc- tional G2 Checkpoint in Irradiated Cells}

MEK2 activation has been reported to be essential for cellular recovery from the $\mathrm{G} 2$ phase arrest and subsequent survival in irradiated HeLa cells. ${ }^{20}$ To study whether the ERK pathway is involved in the cell cycle delay of irradiated cells, cells were pretreated with MEK1/2 inhibitors, either PD 98059 $(50 \mu \mathrm{M})$ or $\mathrm{U} 0126(10 \mu \mathrm{M})$, for $1 \mathrm{hr}$ and then irradiated at $8 \mathrm{~Gy}$ in the presence of these inhibitors. Twenty-four hours after irradiation, the cell cycle distribution of the cells was analyzed (Table 3 ). HeEB5 cells cycled more rapidly after irradiation than EB8-C cells; $62 \%$ of cells had re-entered the G1 phase by $24 \mathrm{hr}$, whereas $83 \%$ of EB8-C cells remained at the G2 phase (see also Table 2). On the other hand, treatment with either PD98059 or U0126 induced delayed recovery from the G2 checkpoint in irradiated HeEB5 cells; 46 and $57 \%$ of cells treated with PD98059 and U0126 stayed at the G2 phase $24 \mathrm{hr}$ after irradiation, respectively. In contrast, treatment with either of these inhibitors did not affect the cell cycle profile in irradiated EB8-C cells.

To further determine whether ROS generated in cells exposed to irradiation affect cell cycle progression, these cells were treated with $25 \mathrm{mM}$ of a free radical scavenger $\mathrm{N}$-acetyl cystein (NAC) for $1 \mathrm{hr}$, and then irradiated at $8 \mathrm{~Gy}$ in the presence of NAC. The cell cycle was analyzed $24 \mathrm{hr}$ after irradiation. NAC treatment also resulted in the delayed recovery from irradiation-induced G2 arrest in HeEB5 cells; $62 \%$ of the cells were at G2/M phase. However, NAC accelerated the recovery slightly in irradiated EB8-C cells. In parallel, the numbers of colonies were counted 2 weeks later. A significantly decreased sensitivity was observed in EB8-C cells; the surviving fractions were $3.3 \pm 0.8 \%$ for HeEB5 cells and $1.6 \pm 0.5 \%$ for EB8-C cells, respectively $(p<0.05)$. Treatment with NAC reduced the surviving fraction in irradiated HeEB5 cells $(0.7 \pm 0.2 \%$, $p<0.05$ ). In contrast, pretreatment with the compound had no significant effect on the fraction of irradiated EB8-C cells $(0.6 \pm 0.5 \%)$. 
Table 3. Effect of MEK1/2 Inhibition and ROS Scavenger on Cell Cycle Progression after Irradiation

\begin{tabular}{llcrrrr}
\hline \hline & & \multicolumn{5}{c}{ Distribution of Cells in Each Stage (\%) } \\
\cline { 3 - 7 } & & Untreated & IR & PD+IR & U+IR & NAC+IR \\
\hline HeEB5 & $\mathrm{G}_{1}$ & 55 & 62 & 46 & 37 & 32 \\
& $\mathrm{~S}$ & 30 & 15 & 8 & 6 & 6 \\
& $\mathrm{G}_{2} / \mathrm{M}$ & 15 & 23 & 46 & 57 & 62 \\
EB8-C & $\mathrm{G}_{1}$ & 56 & 7 & 4 & 7 & 9 \\
& $\mathrm{~S}$ & 31 & 10 & 14 & 12 & 22 \\
& $\mathrm{G}_{2} / \mathrm{M}$ & 13 & 83 & 82 & 81 & 69 \\
\hline
\end{tabular}

Cells were pretreated with either MEK inhibitor PD98059 (PD, $50 \mu \mathrm{M}$, Calbiochem, La Jolla, CA, U.S.A.), U0126 (U, $10 \mu \mathrm{M}$, Cell Signaling Technology, Inc., Beverly, MA, U.S.A.) or a free radical scavenger N-acetyl cysteine (NAC, $25 \mathrm{mM}$, Sigma) for $1 \mathrm{hr}$. Then cells were irradiated with $8 \mathrm{~Gy}$. Twenty-four hr after irradiation, cell cycle analysis with propidium iodide staining was performed. Data are representative of three independent experiments.

\section{DISCUSSION}

ROS are ubiquitously generated at a steady state, and their production is enhanced by irradiation. ${ }^{29)}$ mtDNA is more vulnerable to oxidative stress than nuclear DNA. ${ }^{2}$ Furthermore, mtDNA is continuously replicated even in terminally differentiated cells. It is therefore of major importance that the role(s) of mtDNA in irradiated cells is clarified. In the present study, we compared cells without mtDNA ( $\rho^{0}$ cells, EB8-C) to control cells with intact mtDNA $\left(\rho^{+}\right.$cells, HeEB5) in irradiation. EB8-C cells produced less amounts of ROS than HeEB5 cells both at a steady state and following irradiation. We also showed that EB8-C cells were more sensitive to irradiation than HeEB5 cells, a phenomenon associated with lower post-irradiation ROS production. Irradiated HeEB5 cells exhibited earlier recovery from irradiation-induced G2 arrest, which was blocked by either MEK inhibition or scavenging ROS.

Induction of cell cycle checkpoint responses in cells exposed to irradiation is essential for maintaining genomic integrity by the repair of damaged DNA. ${ }^{26)}$ Oxidative stress such as irradiation causes G1 arrest that is dependent on $\mathrm{p} 53$ activation. ${ }^{26)}$ Since effective G2 arrest and recovery from G2 arrest have also been shown to be essential for the ability of the cell to respond effectively to irradiation, we analyzed the cell cycle profile of HeEB5 and EB8-C cells after irradiation. Following irradiation, HeEB5 cells did not arrest in the G1 phase, entering into the G2 phase. These results were consistent with a previous study reporting that the G2 checkpoint showed arrest due to irradiation damage in HeLa cells. ${ }^{30)}$ On the other hand, the cell cycle progression of irradiated EB8-C cells was delayed, as these cells induced delayed G2 arrest. In addition, HeEB5 cells exited faster from the G2 phase than EB8-C cells, with the latter demonstrating a delayed recovery from the $\mathrm{G} 2$ arrest. Previous studies showed that reconstitution of p53-null cells with functional p53 shortened G2 arrest upon irradiation, ${ }^{31)}$ and that bone marrow cells enriched in normal myeloblasts entered mitosis more frequently than p53-null cells after exposure to irradiation. ${ }^{32,33)}$ Moreover, in ataxia telangiectasia (AT) cells, the DNA damage-dependent G2 arrest is longer than in normal cells, ${ }^{34)}$ and the length of $\mathrm{G} 2$ arrest has been reported to correlate with the radioresistance of the cell. ${ }^{33)}$ These studies suggest that the prolonged G2 phase following DNA damage is due to lower repair efficiency. However, mtDNA does not code for any DNA repair protein. Therefore, our results suggest that activation of mtDNA might lead to initiating a certain signal transduction pathway that protects cells from irradiation. HeEB5 and EB8$\mathrm{C}$ cells originate from HeLa cells. ${ }^{23)} \mathrm{HeLa}$ cells are infected with human papilloma virus and the E6 protein inactivates $\mathrm{p} 53 .{ }^{27)} \mathrm{p} 21^{\text {WAFI }}$ is one of the cyclin dependent kinase (Cdk) inhibitors regulated by p53 and causes cell cycle arrest. ${ }^{35)}$ However, p $21^{\text {WAFI }}$ is also induced by $\mathrm{p} 53$-independednt mechanisms following irradiation. ${ }^{36)}$ Therefore, we studied the expression of $\mathrm{p} 21^{\text {WAFI }}$ in these cells. The $\mathrm{p} 21^{\text {WAFI }}$ expression was not induced by irradiation in HeEB5 and EB8-C cells (data not shown). Our results thus suggest that the increased sensitivity to irradiation and the delayed G2 arrest and delayed recovery from G2 arrest constitute a p53-independent event in these cells.

The MAPK pathways control cell fate in irradiated cells, and ERK signaling is important for radiation sensitivity. ${ }^{16)}$ ROS and irradiation are also known to activate MAPK pathways. ${ }^{6,37,38)}$ Moreover, 
MAPK pathways are necessary for cell cycle progression through G2. ${ }^{39}$ We studied phosphorylation of ERK1/2 in both cell lines irradiated. The phosphorylated form of ERK1/2 was clearly detected in HeEB5 cells and irradiation activated ERK1/2 in these cells. However, irradiation did not activate p38MAPK in both cells (not shown). Moreover, treatment with two different inhibitors specific for MEK1/2 delayed the recovery from G2 arrest in irradiated HeEB5 cells. Interestingly, scavenging ROS with NAC clearly induced the delayed recovery and also increased the radiation sensitivity in these cells. In EB8-C cells, however, irradiation only slightly activated the phosphorylation of ERK1/2, and NAC also induced delayed $\mathrm{G} 2$ arrest, albeit only to a minor extent. However, treatment with these inhibitors did not affect the cell cycle following irradiation in EB8-C cells. Thus, our study showed that the generation of ROS is involved in the regulation of the $\mathrm{G} 2$ checkpoint and that mtDNA is important for increased ROS generation leading to the potentiation of the ERK1/2 pathway to a certain extent in irradiated cells. It has been reported that inhibition of MEK2 upstream of ERK1/2 increased radiosensitivity through a deregulated $\mathrm{G} 2$ checkpoint and that treatment with caffeine reversed the radiosensitivity with a concomitant recovery from the G2 arrest in otherwise terminally arrested HeLa cells with MEK2 mutation. ${ }^{20)}$ In A431 squamous carcinoma cells and DU 145 prostate carcinoma cells, inhibition of MEK1/2 by PD 98059 slowed recovery from the G2/M arrest and enhanced cell death. ${ }^{21,40)}$ Taken together, our results indicate that the efficient activation of the ERK1/2 pathway by the generation of ROS is required for protection of cells from irradiation through the recovery from irradiation-induced G2 arrest in cells. However, mtDNA-depleted cells are unable to activate the ERK pathway because of their disability to effectively generate ROS, whereas NAPDH oxidase existing in cellular membrane and epidermal growth factor-induced production may be source of ROS outside mitochondria ${ }^{10)}$ and ROS production related to cytochrome $\mathrm{c}$ may be also source. ${ }^{41)}$ Thus, it is clear that mtDNA is important for signal transduction as well as oxidative phosphorylation.

mtDNA is a circular, double-stranded molecule encoding 13 proteins that compose part of complex I, III-V (ATP synthase) of the electron transport chain. ${ }^{42)}$ Mammalian mitochondria account for over $90 \%$ of cellular oxygen consumption, and $1-5 \%$ of consumed oxygen is converted to ROS in the mito- chondrial respiratory chain. ${ }^{43)}$ We compared the production of ROS between EB8-C and HeEB5 cells, since the mitochondrial respiratory chain is a powerful source of ROS. HeEB5 cells had a constitutively higher level of ROS with lower activity of glutathione peroxidase (GSH-Px) than EB8-C cells (data not shown). Previous studies by other investigators have also reported decreased generation of ROS in cells lacking mtDNA. ${ }^{9,10,12)}$ We also found greater plating efficiency and larger sizes of colonies in HeEB5 cells than in EB8-C cells. These results indicate that HeEB5 cells have higher growth rate. Our results also suggest that steady-state levels of ROS produced in a regulated fashion may be required for signaling pathways controlling essential cellular function, whereas high levels of ROS may inhibit the activity of cellular components or result in damage and cell death. Thus, mtDNA may play an important role in the generation of ROS that initiate the signal transduction for cell growth.

We studied the role of mtDNA in irradiated cells and showed that EB8-C cells were more sensitive to irradiation. These results are in contrast to those of studies by other investigators, who reported that $\rho^{0}$ cells are resistant to various forms of stress such as oxidative stress, chemicals, TRAIL, and others. ${ }^{6-8,44)}$ The mechanisms responsible for these discrepancies are not clear. One of these studies concluded that up-regulation of manganese superoxide dismutase (MnSOD) and GSH-Px leads to an efficient disposal of increased oxidative stress and increased resistance against ROS in $\rho^{0}$ cells. ${ }^{7}$ In our study, on the other hand, the activity of MnSOD was higher with no difference in that of copper-zinc superoxide dismutase (CuZnSOD) but the activity of GSH-Px was lower in HeEB5 cells, and no difference of catalase (CAT) levels were observed in both cell lines (data not shown). Increased activities of SODs lead to the accumulation of $\mathrm{H}_{2} \mathrm{O}_{2}$ unless the $\mathrm{H}_{2} \mathrm{O}_{2}$ is in turn detoxified by GSH-Px or catalase. Furthermore, our study showed that scavenging ROS with NAC significantly reduced the colony forming capacity in irradiated HeEB5 cells, whereas NAC did not affect that in irradiated EB8-C cells. We could not detect an increase of the ROS generation in cells irradiated with 8 Gy or less in our experiments. However, NAC treatment delayed the recovery from G2 in irradiated HeEB5 cells with 8 Gy and also increased the radiation sensitivity of HeEB5 cells. Our results strongly suggest that mtDNA plays an important role in initiating a certain signal transduction pathway leading to cell survival, whereas the 
role of ROS may vary according to cell type and their concentrations produced.

Acknowledgements This work is supported in part by a Grant-in-Aid for Science Research from the Japan Society for the Promotion of Science (JSPS) (No. 16591242) and also a research project of the Radiation Emergency Medical Preparedness by National Institute of Radiological Sciences. We would like to thank Ms. Aki Yamamoto and Ms. Kyoko Takayama for their secretarial assistance.

\section{REFFERENCES}

1) Saraste, M. (1999) Oxidative phosphorylation at the fin de siecle. Science, 283, 1488-1493.

2) Brown, W. M., George, M., Jr. and Wilson, A. C. (1979) Rapid evolution of animal mitochondrial DNA. Proc. Natl. Acad. Sci. U.S.A., 76, 1967-1971.

3) Tan, D. J., Bai, R. K. and Wong, L. J. (2002) Comprehensive scanning of somatic mitochondrial DNA mutations in breast cancer. Cancer Res., 62, 972976.

4) Escary, J. L., Perreau, J., Dumenil, D., Ezine, S. and Brulet, P. (1993) Leukaemia inhibitory factor is necessary for maintenance of haematopoietic stem cells and thymocyte stimulation. Nature (London), 363, 361-364.

5) Marchetti, P., Susin, S. A., Decaudin, D., Gamen, S., Castedo, M., Hirsch, T., Zamzami, N., Naval, J., Senik, A. and Kroemer, G. (1996) Apoptosis-associated derangement of mitochondrial function in cells lacking mitochondrial DNA. Cancer Res., 56, 2033-2038.

6) Jiang, S., Cai, J., Wallace, D. C. and Jones, D. P. (1999) Cytochrome c-mediated apoptosis in cells lacking mitochondrial DNA. Signaling pathway involving release and caspase 3 activation is conserved. J. Biol. Chem., 274, 29905-29911.

7) Park, S. Y., Chang, I., Kim, J. Y., Kang, S. W., Park, S. H., Singh, K. and Lee, M. S. (2004) Resistance of mitochondrial DNA-depleted cells against cell death: role of mitochondrial superoxide dismutase. J. Biol. Chem., 279, 7512-7520.

8) Kim, J. Y., Kim, Y. H., Chang, I., Kim, S., Pak, Y. K., Oh, B. H., Yagita, H., Jung, Y. K., Oh, Y. J. and Lee, M. S. (2002) Resistance of mitochondrial DNAdeficient cells to TRAIL: role of Bax in TRAILinduced apoptosis. Oncogene, 21, 3139-3148.

9) Li, N., Ragheb, K., Lawler, G., Sturgis, J., Rajwa, B., Melendez, J. A. and Robinson, J. P. (2003) Mitochondrial complex I inhibitor rotenone induces apoptosis through enhancing mitochondrial reactive oxygen species production. J. Biol. Chem., 278, 8516-8525.

10) Walford, G. A., Moussignac, R. L., Scribner, A. W., Loscalzo, J. and Leopold, J. A. (2003) Hypoxia potentiates nitric oxide-mediated apoptosis in Endothelial cells via peroxynitrite-induced activation of mitochondria-dependent and -independent pathways. J. Biol. Chem., 279, 4425-4432.

11) Sidoti-de Fraisse, C., Rincheval, V., Risler, Y., Mignotte, B. and Vayssiere, J. L. (1998) TNF-alpha activates at least two apoptotic signaling cascades. Oncogene, 17, 1639-1651.

12) Leach, J. K., Van Tuyle, G., Lin, P. S., SchmidtUllrich, R. and Mikkelsen, R. B. (2001) Ionizing radiation-induced, mitochondria-dependent generation of reactive oxygen/nitrogen. Cancer Res., 61, 3894-3901.

13) Nemoto, S., Takeda, K., Yu, Z. X., Ferrans, V. J. and Finkel, T. (2000) Role for mitochondrial oxidants as regulators of cellular metabolism. Mol. Cell. Biol., 20, 7311-7318.

14) Waypa, G. B., Chandel, N. S. and Schumacker, P. T. (2001) Model for hypoxic pulmonary vasoconstriction involving mitochondrial oxygen sensing. Circ. Res., 88, 1259-1266.

15) Nishikawa, T., Edelstein, D., Du, X. L., Yamagishi, S., Matsumura, T., Kaneda, Y., Yorek, M. A., Beebe, D., Oates, P. J., Hammes, H. P., Giardino, I. and Brownlee, M. (2000) Normalizing mitochondrial superoxide production blocks three pathways of hyperglycaemic damage. Nature (London), 404, 787-790.

16) Dent, P., Yacoub, A., Fisher, P. B., Hagan, M. P. and Grant, S. (2003) MAPK pathways in radiation responses. Oncogene, 22, 5885-5896.

17) Shonai, T., Adachi, M., Sakata, K., Takekawa, M., Endo, T., Imai, K. and Hareyama, M. (2002) MEK/ ERK pathway protects ionizing radiation-induced loss of mitochondrial membrane potential and cell death in lymphocytic leukemia cells. Cell Death Differ., 9, 963-971.

18) Tran, S. E., Holmstrom, T. H., Ahonen, M., Kahari, V. M. and Eriksson, J. E. (2001) MAPK/ERK overrides the apoptotic signaling from Fas, TNF, and TRAIL receptors. J. Biol. Chem., 276, 16484-16490.

19) Yang, S. H., Sharrocks, A. D. and Whitmarsh, A. J. (2003) Transcriptional regulation by the MAP kinase signaling cascades. Gene, 320, 3-21.

20) Abbott, D. W. and Holt, J. T. (1999) Mitogen-activated protein kinase kinase 2 activation is essential for progression through the G2/M checkpoint arrest in cells exposed to ionizing radiation. J. Biol. Chem., 274, 2732-2742. 
21) Hagan, M., Wang, L., Hanley, J. R., Park, J. S. and Dent, P. (2000) Ionizing radiation-induced mitogenactivated protein (MAP) kinase activation in DU145 prostate carcinoma cells: MAP kinase inhibition enhances radiation-induced cell killing and G2/Mphase arrest. Radiat. Res., 153, 371-383.

22) King, M. P. and Attardi, G. (1989) Human cells lacking mtDNA: repopulation with exogenous mitochondria by complementation. Science, 246, 500-503.

23) Hayashi, J., Ohta, S., Kikuchi, A., Takemitsu, M., Goto, Y. and Nonaka, I. (1991) Introduction of disease-related mitochondrial DNA deletions into HeLa cells lacking mitochondrial DNA results in mitochondrial dysfunction. Proc. Natl. Acad. Sci. U.S.A., 88, 10614-10618.

24) Bradford, M. M. (1976) A rapid and sensitive method for the quantitation of microgram quantities of protein utilizing the principle of protein-dye binding. Anal. Biochem., 72, 248-254.

25) Morales, A., Miranda, M., Sanchez-Reyes, A., Biete, A. and Fernandez-Checa, J. C. (1998) Oxidative damage of mitochondrial and nuclear DNA induced by ionizing radiation in human hepatoblastoma cells Int. J. Radiat. Oncol. Biol. Phys., 42, 191-203.

26) Shackelford, R. E., Kaufmann, W. K. and Paules, R. S. (2000) Oxidative stress and cell cycle checkpoint function. Free Radic. Biol. Med., 28, 13871404.

27) Werness, B. A., Levine, A. J. and Howley, P. M. (1990) Association of human papillomavirus types 16 and 18 E6 proteins with p53. Science, 248, 7679 .

28) Takada, Y., Hachiya, M., Park, S. H., Osawa, Y., Ozawa, T. and Akashi, M. (2002) Role of reactive oxygen species in cells overexpressing manganese superoxide dismutase: mechanism for induction of radioresistance. Mol. Cancer Res., 1, 137-146.

29) Yang, Y. and Yu, X. (2003) Regulation of apoptosis: the ubiquitous way. FASEB J., 17, 790-799.

30) Maity, A., Kao, G. D., Muschel, R. J. and McKenna, W. G. (1997) Potential molecular targets for manipulating the radiation response. Int. J. Radiat. Oncol. Biol. Phys., 37, 639-653.

31) Schwartz, D., Almog, N., Peled, A., Goldfinger, N. and Rotter, V. (1997) Role of wild type p53 in the G2 phase: regulation of the gamma-irradiation-induced delay and DNA repair. Oncogene, 15, 25972607.

32) Lavin, M. F. and Shiloh, Y. (1997) The genetic defect in ataxia-telangiectasia. Annu. Rev. Immunol., 15, 177-202.

33) Aldridge, D. R. and Radford, I. R. (1998) Explaining differences in sensitivity to killing by ionizing radiation between human lymphoid cell lines. Cancer Res., 58, 2817-2824.

34) Hawley, R. S. and Friend, S. H. (1996) Strange bedfellows in even stranger places: the role of ATM in meiotic cells, lymphocytes, tumors, and its functional links to p53. Genes Dev., 10, 2383-2388.

35) Gartel, A. L. and Tyner, A. L. (2002) The role of the cyclin-dependent kinase inhibitor p21 in apoptosis. Mol. Cancer Ther., 1, 639-649.

36) Akashi, M., Hachiya, M., Osawa, Y., Spirin, K., Suzuki, G. and Koeffler, H. P. (1995) Irradiation induces WAF1 expression through a p53-independent pathway in KG-1 cells. J. Biol. Chem., 270, 19181-19187.

37) Torres, M. (2003) Mitogen-activated protein kinase pathways in redox signaling. Front Biosci., 8, 369391.

38) Zhang, Z., Leonard, S. S., Huang, C., Vallyathan, V., Castranova, V. and Shi, X. (2003) Role of reactive oxygen species and MAPKs in vanadate-induced G(2)/M phase arrest. Free Radic. Biol. Med., 34, 1333-1342.

39) Abrieu, A., Doree, M. and Picard, A. (1997) Mitogen-activated protein kinase activation down-regulates a mechanism that inactivates cyclin B-cdc2 kinase in G2-arrested oocytes. Mol. Biol. Cell., 8, 249-261.

40) Park, J. S., Carter, S., Reardon, D. B., SchmidtUllrich, R., Dent, P. and Fisher, P. B. (1999) Roles for basal and stimulated p21(Cip-1/WAF1/MDA6) expression and mitogen-activated protein kinase signaling in radiation-induced cell cycle checkpoint control in carcinoma cells. Mol. Biol. Cell., 10 , 4231-4246.

41) Mikkelsen, R. B. and Wardman, P. (2003) Biological chemistry of reactive oxygen and nitrogen and radiation-induced signal transduction mechanisms. Oncogene, 22, 5734-5754.

42) Anderson, S., Bankier, A. T., Barrell, B. G., de Bruijn, M. H., Coulson, A. R., Drouin, J., Eperon, I. C., Nierlich, D. P., Roe, B. A., Sanger, F., Schreier, P. H., Smith, A. J., Staden, R. and Young, I. G. (1981) Sequence and organization of the human mitochondrial genome. Nature (London), 290, 457-465.

43) Boveris, A. and Chance, B. (1973) The mitochondrial generation of hydrogen peroxide. General properties and effect of hyperbaric oxygen. Biochem. J., 134, 707-716.

44) Dey, R. and Moraes, C. T. (2000) Lack of oxidative phosphorylation and low mitochondrial membrane potential decrease susceptibility to apoptosis and do not modulate the protective effect of $\mathrm{Bcl}-\mathrm{x}(\mathrm{L})$ in osteosarcoma cells. J. Biol. Chem., 275, 7087-7094. 